\title{
Justificativa e Desenho do Ensaio Clínico Randomizado COVID-19 Outpatient Prevention Evaluation (COPE - Coalition V): Hidroxicloroquina vs. Placebo em Pacientes Não Hospitalizados
}

\author{
Rationale and Design of the COVID-19 Outpatient Prevention Evaluation (COPE - Coalition V) Randomized \\ Clinical Trial: Hydroxychloroquine vs. Placebo in Non-Hospitalized Patients
}

\author{
Haliton Alves de Oliveira Junior, ${ }^{1 \oplus}$ Cleusa P. Ferri, ${ }^{1}$ Icaro Boszczowski, ${ }^{1}$ Gustavo B. F. Oliveira, ${ }^{10}$ \\ Alexandre B. Cavalcanti, ${ }^{2,3}$ Regis G. Rosa, ${ }^{3,4}$ Renato D. Lopes, 5,6 Luciano C. P. Azevedo, ${ }^{3,7}$ Viviane C. Veiga, ${ }^{3,8}$ \\ Otavio Berwanger, ${ }^{9}$ Álvaro Avezum, ${ }^{1}$ em nome dos Investigadores do COPE - COALIZÃO COVID-19 V \\ Centro Internacional de Pesquisa, Hospital Alemão Oswaldo Cruz, ${ }^{1}$ São Paulo, SP - Brasil \\ Instituto de Pesquisa HCor, ${ }^{2}$ São Paulo, SP - Brasil \\ Brazilian Research in Intensive Care Network (BRICnet), ${ }^{3}$ São Paulo, SP - Brasil \\ Hospital Moinhos de Vento, ${ }^{4}$ Porto Alegre, PR - Brasil \\ Brazilian Clinical Research Institute (BCRI), ${ }^{5}$ São Paulo, SP - Brasil \\ Duke University Medical Center - Duke Clinical Research Institute, ${ }^{6}$ Durham - EUA \\ Instituto de Ensino e Pesquisa do Hospital Sírio-Libanês, 7 São Paulo, SP - Brasil \\ Beneficência Portuguesa de São Paulo, ${ }^{8}$ São Paulo, SP - Brasil \\ Academic Research Organization, Hospital Israelita Albert Einstein, ${ }^{9}$ São Paulo, SP - Brasil
}

\section{Resumo}

Fundamento: Apesar da necessidade de opções terapêuticas específicas para a doença do coronavírus 2019 (covid-19), ainda não há evidências da eficácia de tratamentos específicos no contexto ambulatorial. Há poucos estudos randomizados que avaliam a hidroxicloroquina (HCQ) em pacientes não hospitalizados. Esses estudos não indicaram benefício com o uso da HCQ; no entanto, avaliaram desfechos primários diferentes e apresentaram vieses importantes na avaliação dos desfechos.

Objetivo: Investigar se a HCQ possui o potencial de prevenir hospitalizações por covid-19 quando comparada ao placebo correspondente.

Métodos: O estudo COVID-19 Outpatient Prevention Evaluation (COPE) é um ensaio clínico randomizado, pragmático, duplo-cego, multicêntrico e controlado por placebo que avalia o uso da HCQ (800 mg no dia 1 e $400 \mathrm{mg}$ do dia 2 ao dia 7) ou placebo correspondente na prevenção de hospitalizações por covid-19 em casos precoces confirmados ou suspeitos de pacientes não hospitalizados. Os critérios de inclusão são adultos ( $\geq 18$ anos) que procuraram atendimento médico com sintomas leves de covid-19, com randomização $\leq 7$ dias após o início dos sintomas, sem indicação de hospitalização na triagem do estudo e com pelo menos um fator de risco para complicações ( $>65$ anos, hipertensão, diabetes melito, asma, doença pulmonar obstrutiva crônica ou outras doenças pulmonares crônicas, tabagismo, imunossupressão ou obesidade). Todos os testes de hipótese serão bilaterais. Um valor de $p<0,05$ será considerado estatisticamente significativo em todas as análises. Clinicaltrials.gov: NCT04466540.

Resultados: Os desfechos clínicos serão avaliados centralmente por um comitê de eventos clínicos independente cegado para a alocação dos grupos de tratamento. O desfecho primário de eficácia será avaliado de acordo com o princípio da intenção de tratar.

Conclusão: Este estudo apresenta o potencial de responder de forma confiável a questão científica do uso da HCQ em pacientes ambulatoriais com covid-19. Do nosso conhecimento, este é o maior estudo avaliando o uso de HCQ em indivíduos com covid-19 não hospitalizados.

Palavras-chave: COVID-19; SARS-CoV-2; Hidroxicloroquina; Ensaio Clínico Controlado Aleatório.

Correspondência: Álvaro Avezum •

Hospital Alemão Oswaldo Cruz - International Research Center - Rua João Julião, 331. CEP 01323-903, Sao Paulo, SP - Brasil

E-mail: aavezum@haoc.com.br

Artigo recebido em 28/09/2021, revisado em 12/11/2021, aceito em 24/11/2021

DOI: https://doi.org/10.36660/abc.20210832 


\begin{abstract}
Background: Despite the need for targeting specific therapeutic options for coronavirus disease 2019 (COVID-19), there has been no evidence of effectiveness of any specific treatment for the outpatient clinical setting. There are few randomized studies evaluating hydroxychloroquine (HCQ) in non-hospitalized patients. These studies indicate no benefit from the use of HCQ, but they assessed different primary outcomes and presented important biases for outcome evaluation.
\end{abstract}

Objective: To evaluate if HCQ may prevent hospitalization due to COVID-19 compared to a matching placebo.

Methods: The COVID-19 Outpatient Prevention Evaluation (COPE) study is a pragmatic, randomized, double-blind, placebo-controlled clinical trial evaluating the use of HCQ (800 mg on day 1 and $400 \mathrm{mg}$ from day 2 to day 7) or matching placebo for the prevention of hospitalization due to COVID-19 in early non-hospitalized confirmed or suspected cases. Inclusion criteria are adults ( $\geq 18$ years) seeking medical care with mild symptoms of COVID-19, with randomization $\leq 7$ days after symptom onset, without indication of hospitalization at study screening, and with at least one risk factor for complication (> 65 years; hypertension; diabetes mellitus; asthma; chronic obstructive pulmonary disease or other chronic lung diseases; smoking; immunosuppression; or obesity). All hypothesis tests will be two-sided. A p-value $<0.05$ will be considered statistically significant in all analyses. Clinicaltrials.gov: NCT04466540.

Results: Clinical outcomes will be centrally adjudicated by an independent clinical event committee blinded to the assigned treatment groups. The primary efficacy endpoint will be assessed following the intention-to-treat principle.

Conclusion: This study has the potential to reliably answer the scientific question of HCQ use in outpatients with COVID-19. To our knowledge, this is the largest trial evaluating HCQ in non-hospitalized individuals with COVID-19.

Keywords: COVID-19; SARS-CoV-2; Hydroxychloroquine; Randomized Controlled Trial.

Full texts in English - https://abccardiol.org/en/

\section{Introdução}

Em dezembro de 2019, um grupo de pacientes com pneumonia de causa desconhecida foi identificado em Wuhan, na província de Hubei, na China. ${ }^{1}$ O sequenciamento de alto rendimento de amostras do trato respiratório inferior indicou a presença de um novo coronavírus, chamado novo coronavírus 2019 ou, mais recentemente, coronavírus ${ }^{2}$ causador da síndrome respiratório aguda grave (SARS-CoV-2), que causa uma condição clínica complicada que afeta a função pulmonar (denominada doença do coronavírus 2019, ou covid-19), a qual ainda não havia sido detectada em seres humanos ou animais. ${ }^{1-4}$

Apesar da necessidade de terapias específicas para a covid-19, não há evidências claras da efetividade de nenhum tratamento no contexto ambulatorial. Dessa forma, a avaliação de opções terapêuticas, como agentes farmacológicos com propriedades antivirais, é essencial para reduzir o risco de deterioração clínica, hospitalização, necessidade de ventilação mecânica e morte, especialmente na fase inicial da covid-19 no contexto ambulatorial. Atualmente, há alguns ensaios clínicos randomizados (ECRs) avaliando o efeito da cloroquina/ hidroxicloroquina (HCQ) em pacientes não hospitalizados. No contexto da profilaxia pré e pós-exposição, os estudos clínicos não identificaram benefícios em relação à taxa de infecção da covid-19, ${ }^{5-9}$ enquanto outros estudos identificaram um aumento na ocorrência de efeitos adversos em pacientes que receberam cloroquina/HCQ. ${ }^{6,7}$ Contudo, é importante ressaltar que esses estudos continham vieses significativos e, quando considerados em conjunto, apresentaram heterogeneidade significativa de resultados devido a diferenças nos regimes de dosagem, critérios de inclusão e desfechos primários.

Considerando os casos de covid-19 não hospitalizados, os ECRs não encontraram diferenças significativas na taxa de hospitalização ao comparar HCQ e placebo $^{10}$ ou cuidado usual. ${ }^{11}$ Além disso, alguns ECRs não identificaram benefício em relação à cura ou redução da carga viral ao comparar HCQ e placebo ${ }^{12}$ ou cuidado usual. ${ }^{10}$ Alguns ECRs relataram um aumento na ocorrência de eventos adversos em pacientes que receberam HCQ. ${ }^{10,11}$ Portanto, são necessários estudos mais abrangentes, com melhor rigor metodológico.

O principal objetivo do presente estudo é investigar se o tratamento precoce com HCQ diminui o risco de hospitalização (desfecho primário de eficácia) por complicações clínicas relacionadas à covid-19 em 30 dias após a randomização.

\section{Métodos}

\section{Desenho do estudo}

Este é um ensaio clínico randomizado, pragmático, duplo-cego, multicêntrico e controlado por placebo. Foi realizada alocação na proporção 1:1 para avaliar as potenciais propriedades antivirais do tratamento precoce com HCQ (800 mg VO no dia 1 e $400 \mathrm{mg}$ VO do dia 2 ao dia 7) em comparação com placebo correspondente na prevenção de hospitalizações por complicações relacionadas à covid-19 em pacientes não hospitalizados com suspeita ou confirmação de covid-19. O fluxo de trabalho planejado é apresentado na Figura 1. O presente estudo segue os Standard Protocol Items: Recommendations for Interventional Trials (SPIRIT) (Tabela Suplementar 1).

\section{Local do estudo}

Este estudo será conduzido em 56 centros de diferentes regiões geográficas do Brasil. Os centros são serviços de saúde ambulatoriais privados e públicos que receberam aprovação para participar do estudo após avaliação de viabilidade favorável, conformidade com as boas práticas clínicas e aprovação do comitê de ética. 


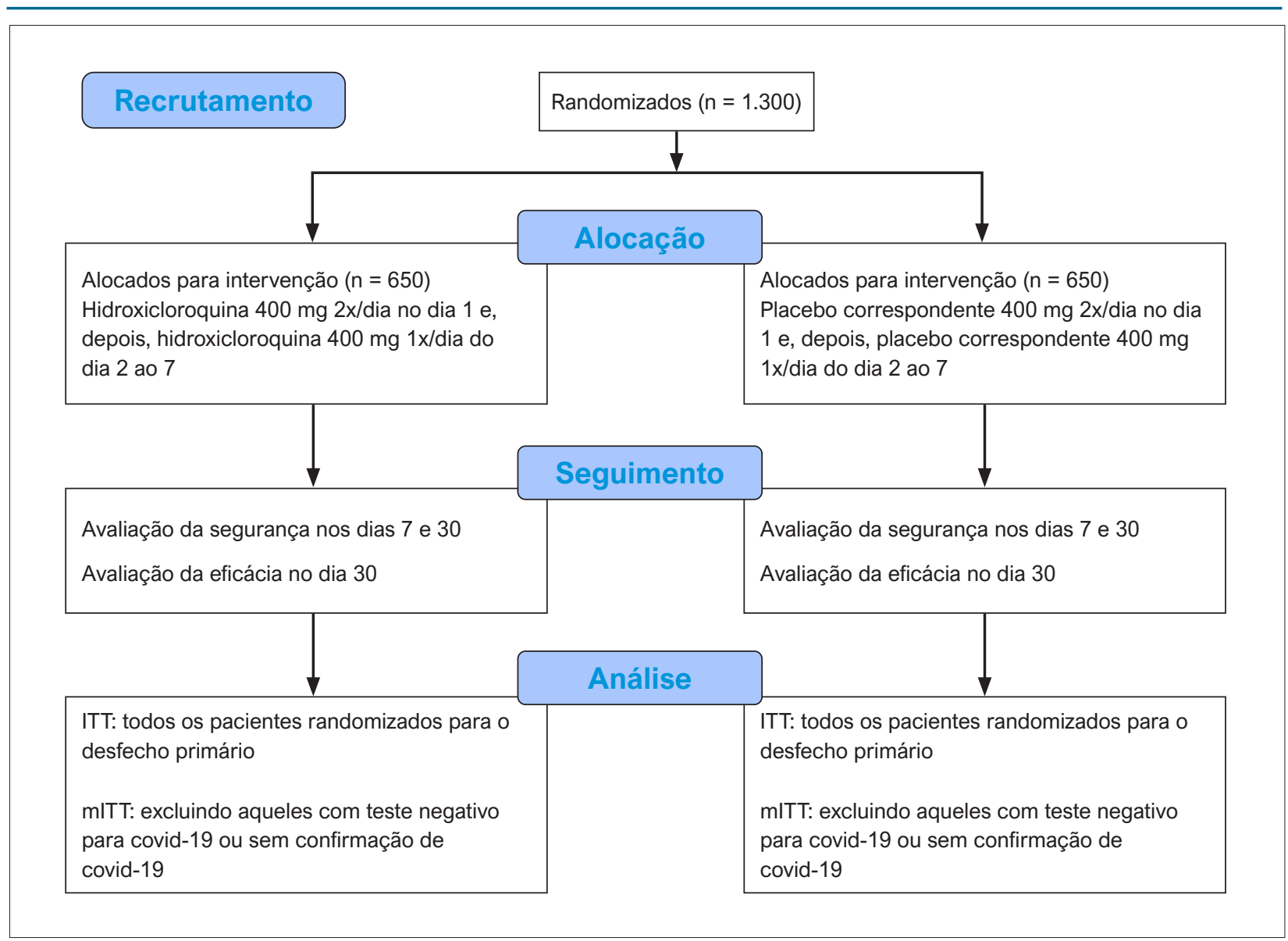

Figura 1 - Diagrama CONSORT mostrando o fluxo de trabalho e o recrutamento planejado para o estudo. ITT: intenção de tratar; mITT: intenção de tratar modificada.

\section{Objetivo primário}

Objetivamos investigar se o tratamento com HCQ está associado a uma redução na necessidade de hospitalização por complicações clínicas relacionadas à covid-19 suspeita ou confirmada em 30 dias após a randomização no contexto ambulatorial. A hospitalização é definida como um período de permanência hospitalar $\geq 24$ horas ou pelo menos mais um dia de hospitalização adjudicada. Os critérios de hospitalização seguirão a prática clínica local de cada centro participante.

\section{Objetivos secundários}

Avaliar o efeito do tratamento com HCQ comparado ao placebo em pacientes ambulatoriais com suspeita ou confirmação de covid-19, aos 30 dias de seguimento, em relação aos seguintes desfechos:

1. Asma não controlada após $\geq 5$ dias desde o início do medicamento: resposta afirmativa em três ou quatro itens do questionário Global Initiative for Asthma, descrito na Tabela Suplementar 2;

2. Pneumonia: definida por critérios clínico-radiológicos, incluindo histórico de tosse e a presença de um ou mais dos seguintes sinais/sintomas: escarro, dispneia, dor no peito, suor ou febre $(>37,80 \mathrm{C})+$ tomografia computadorizada do tórax com opacidade em vidro fosco uni ou bilateral, consolidações focais ou opacidades mistas (incluindo sinal do halo invertido);

3. Otite média: definida por critérios clínicos de febre (>37,8o C) e otalgia + protuberância da membrana timpânica;

4. Tempo até a resolução da febre: o dia 0 da resolução da febre será definido como o primeiro dia sem febre $(<37,5$ o C ) após a inclusão no estudo, seguido por pelo menos 2 dias consecutivos. A temperatura será obtida através de relato do participante no diário do paciente;

5. Tempo até a melhora dos sintomas respiratórios (tosse, coriza);

6. Hospitalização em unidade de terapia intensiva (UTI): admissão na UTI por complicações clínicas relacionadas à covid-19;

7. Necessidade de intubação orotraqueal: necessidade clínica conforme a avaliação do médico assistente;

8. Duração da ventilação mecânica: número de dias na ventilação mecânica até a extubação ou morte; 
9. Mortalidade: morte por qualquer causa que ocorra em 30 dias após a randomização;

A segurança clínica também será avaliada em 30 dias após a randomização:

1. Hipoglicemia: alteração na frequência de episódios em pacientes diabéticos em uso de hipoglicemiantes, percebida por sinais ou sintomas clínicos ou medida em dispositivos de glicemia capilar ou venosa;

2. Palpitações: autopercepção de batimentos cardíacos que poderiam ser diagnosticados como arritmias em pacientes sem histórico conhecido de intervalo QTC prolongado ou doença cardíaca preexistente;

3. Acuidade visual reduzida: alteração na acuidade visual ou diagnóstico recente de retinopatia não documentada anteriormente;

4. Diarreia: alteração no hábito intestinal com três ou mais episódios diarreicos por dia durante o uso de HCQ e 3 dias após o término do tratamento;

5. Anorexia: alteração no apetite durante o uso de HCQ e 3 dias após o término do tratamento;

6. Labilidade emocional: percepção de alteração na labilidade emocional (oscilações de humor) durante o uso de HCQ e 3 dias após o término do tratamento.

\section{Objetivos exploratórios}

Avaliar o efeito do tratamento com HCQ comparado ao placebo em pacientes ambulatoriais com suspeita ou confirmação de covid-19 em relação aos seguintes desfechos:

1. Tempo até a hospitalização após a randomização;
2. Avaliação do estado clínico do paciente no momento da hospitalização.

\section{Critérios de elegibilidade}

Definição de caso de covid-19:

- A avaliação clínica inicial dos pacientes e a triagem de elegibilidade para o estudo serão realizadas com base na classificação de casos confirmados e suspeitos, desenvolvida de acordo com as diretrizes do Ministério da Saúde e as recomendações da Organização Mundial da Saúde ${ }^{13,14}$ sobre a definição de casos, adaptada para o contexto ambulatorial (Tabela 1). Os critérios de exclusão e inclusão estão descritos na Tabela 2, incluindo aqueles relacionados à segurança cardiovascular, visto que a HCQ pode prolongar o intervalo QTC. ${ }^{15}$

\section{Método de randomização e alocação oculta}

A randomização $(1: 1)$ será gerada por um software on-line e realizada em blocos de permutação de tamanho 8. A ocultação da lista de randomização é mantida por meio de um sistema de randomização centralizado, automatizado e disponível 24 horas on-line.

\section{Cegamento}

Os pacientes, pesquisadores e profissionais de saúde serão cegados para a alocação dos medicamentos. Os desfechos clínicos serão avaliados de forma cega pelo Comitê de Julgamento de Eventos Clínicos.

Tabela 1 - Definição de caso de covid-19 para o estudo COPE (COALITION V), adaptado de acordo com as diretrizes do Ministério da Saúde e da Organização Mundial da Saúde

\section{Caso de covid-19 Definição \\ - Indivíduos com confirmação laboratorial de covid-19 (detecção por RT-PCR do vírus SARS-CoV-2), com amostra coletada preferencialmente entre $04^{\circ}$ e $07^{\circ}$ dia desde 0 início dos sintomas por swabs nasofaríngeos/orofaríngeos, independentemente dos sinais e sintomas.}

Confirmado

- Teste imunológico (teste rápido ou sorologia clássica para detecção de anticorpos lgM/lgG) em amostra coletada a partir do $7^{\circ}$ dia desde 0 início dos sintomas e analisada por método validado.

Pacientes que atendem a pelo menos um dos seguintes critérios*:

- $\quad$ Pacientes com doença respiratória aguda (febre E pelo menos um sinal/sintoma de doença respiratória, como tosse ou Suspeito dispneia) E histórico de viagem ou residência em local com relato de transmissão comunitária de covid-19 nos 14 dias anteriores ao início dos sintomas;

- Pacientes com doença respiratória aguda E contato com um caso confirmado ou provável (com doença respiratória aguda sem confirmação laboratorial) de covid-19 nos 14 dias anteriores ao início dos sintomas.

\footnotetext{
* Dependendo do estado clínico do paciente, esses critérios podem ser complementados por achados radiológicos (infiltração intersticial na radiografia do tórax e/ou opacidade em vidro fosco na tomografia computadorizada do pulmão). É importante ressaltar que a maioria dos pacientes a serem tratados apresentarão sintomas leves, sem indicação clínica para exame de imagem.
} 

sintomas, com sintomas leves, sem indicação clara de hospitalização e pelo menos um dos seguintes fatores de risco para complicações clínicas:

\section{Critérios de inclusão}

\begin{tabular}{ll}
\hline 1. & Idade $>65$ anos; \\
\hline 2. & Hipertensão; \\
\hline 3. & Diabetes melito; \\
\hline 4. & Asma; \\
\hline 5. & Doença pulmonar obstrutiva crônica ou outras doenças pulmonares crônicas; \\
\hline 6. & Tabagismo; \\
\hline 7. & Imunossupressão; \\
\hline 8. & Obesidade (definida como índice de massa corporal $\geq 30 \mathrm{~kg} / \mathrm{m} 2)$. \\
\hline
\end{tabular}

Critérios de exclusão

\begin{tabular}{|c|c|}
\hline 1. & Hospitalização imediata após o primeiro atendimento médico; \\
\hline 2. & Teste positivo para influenza no primeiro atendimento médico; \\
\hline 3. & Hipersensibilidade conhecida à hidroxicloroquina/cloroquina; \\
\hline 4. & Diagnóstico prévio de retinopatia ou degeneração macular; \\
\hline 5. & $\begin{array}{l}\text { Diagnóstico prévio de síndrome do QT longo, histórico de morte súbita em parentes próximos (pais e irmãos), insuficiência } \\
\text { cardíaca descompensada, doença arterial coronariana instável, uso de medicamentos antiarrítmicos ou outros medicamentos } \\
\text { que possam aumentar a biodisponibilidade ou o efeito da hidroxicloroquina; }\end{array}$ \\
\hline 6. & Evidência de doença hepática conhecida, relatada pelo paciente; \\
\hline 7. & Evidência de doença renal crônica conhecida, relatada pelo paciente; \\
\hline 8. & Pacientes com pancreatite; \\
\hline 9 & Eletrocardiograma basal com intervalo QTc $\geq 480 \mathrm{~ms}$; \\
\hline 10. & Uso crônico de hidroxicloroquina/cloroquina por outros motivos; \\
\hline & Gravidez. \\
\hline
\end{tabular}

\section{Intervenções do estudo}

Os dois braços do estudo receberão cuidado usual de acordo com a prática local, que basicamente consiste em recomendações gerais e medicação para alívio dos sintomas. As medidas de apoio e cuidado padrão são definidas como quaisquer tratamentos além dos medicamentos utilizados no estudo necessários para o cuidado do paciente com covid-19, a critério do médico assistente.

Os pacientes do grupo HCQ receberão uma dose de $400 \mathrm{mg} 2 \mathrm{x} /$ dia no primeiro dia e uma dose de $400 \mathrm{mg}$ 1x/dia do segundo ao sétimo dia. Os pacientes do grupo placebo receberão o mesmo regime de administração.

\section{Avaliação dos desfechos e seguimento}

Serão realizados dois contatos por telefone com os participantes (7 e 30 dias) para avaliar a adesão, os sintomas e a necessidade de atendimento médico para detectar uma possível progressão da doença ou a presença de eventos adversos causados pelo tratamento.

\section{Interrupção do tratamento}

Caso sejam confirmados testes negativos para covid-19 em pacientes com suspeita de infecção, o seguinte procedimento será realizado:

- Quando o teste para SARS-CoV-2 (reação da transcriptase reversa seguida pela reação em cadeia da polimerase/reverse transcription polymerase chain reaction, RT-PCR) for realizado no hospital em que o paciente foi randomizado, o pesquisador responsável do centro deverá obter essa informação e compartilhála com o centro coordenador (Centro Internacional de Pesquisa do Hospital Alemão Oswaldo Cruz). O paciente será aconselhado a interromper o tratamento e continuará sendo acompanhado até o fim do seguimento de 30 dias.

- Quando o teste para SARS-CoV-2 (RT-PCR) for realizado em outro laboratório, o participante deverá entrar em contato com o centro em que foi randomizado, o qual, então, deverá informar o centro coordenador. 
O paciente será aconselhado a interromper o tratamento ou não, conforme apropriado, e continuará sendo acompanhado por até 30 dias.

O estudo será interrompido caso sejam observados benefícios claros da intervenção no desfecho primário ou um aumento na frequência de eventos adversos graves. O Comitê de Monitoramento de Segurança de Dados (Data Safety Monitoring Board, DSMB) irá monitorar atentamente qualquer ocorrência de eventos adversos imprevistos ou graves para, se necessário, recomendar o encerramento do estudo para garantir a segurança dos pacientes.

\section{Relato e manejo de eventos adversos}

Neste protocolo, os eventos adversos não são considerados um desfecho do estudo, exceto aqueles classificados como graves (hospitalização por covid-19 e morte).

A coleta ativa dos eventos adversos ocorrerá a partir do momento em que o participante assinar o Termo de Consentimento Livre e Esclarecido (TCLE). As informações coletadas devem incluir os dados do histórico clínico do paciente e as comorbidades, o diagnóstico do evento (com base em sinais e sintomas), a classificação da gravidade, a data de início, a definição da probabilidade de relação causal, bem como a causa do evento de acordo com o pesquisador, a decisão médica, a evolução do paciente em relação aos desfechos adversos, os critérios utilizados para classificar a gravidade do evento e a data de término.

\section{Relato e julgamento dos desfechos}

O desfecho primário será avaliado por médicos com experiência prévia e atual na validação de eventos clínicos com base em padrões internacionais. Hospitalizações em 30 dias por causas relacionadas à covid-19 serão documentadas pela equipe médica. As informações serão coletadas para análise pelo Comitê de Julgamento de Eventos Clínicos, sob alocação confidencial (cegado para a avaliação de eventos clínicos), conforme critérios padronizados. O DSMB avaliará os efeitos da HCQ em comparação ao tratamento com placebo para a medida de desfecho primário (hospitalização em 30 dias) e para eventos adversos (ocorridos em 7 e 30 dias) com necessidade de atendimento médico e/ou hospitalização.

\section{Coleta e gerenciamento de dados}

Os dados serão coletados através de uma ficha clínica (electronic case report form, eCRF) disponível on-line e serão inseridos na eCRF por cada centro participante. O treinamento e o suporte para a utilização do sistema serão disponibilizados aos pesquisadores pelo centro coordenador.

Os dados serão coletados diretamente do paciente e/ ou da família. Diversos procedimentos serão aplicados para garantir a qualidade dos dados (Tabela 3). Os dados a serem coletados durante as visitas incluem:

1. Admissão (início do estudo):

a. Idade, sexo, estado civil, etnia, nível educacional, renda familiar e comorbidades;

b. Resultados de testes moleculares ou sorológicos para covid-19 (dependendo do tempo desde o início dos sintomas/diagnóstico clínico);

c. Uso concomitante de medicamentos no início do estudo;

d. Duração dos sintomas;

2. No sétimo dia após a randomização:

a. Avaliação da segurança (monitoramento de eventos adversos);

b. Adesão ao medicamento;

3. No 30ำ dia após a randomização:

a. Avaliação de eficácia (necessidade de hospitalização);

b. Avaliação de segurança (monitoramento de eventos adversos);

O esquema da coleta de dados e do seguimento dos participantes é apresentado na Figura 2.

Tabela 3 - Passos para garantir a qualidade da coleta e do gerenciamento dos dados

\begin{tabular}{ll}
\hline Item & Definição \\
\hline Visita de iniciação & $\begin{array}{l}\text { Todos os pesquisadores participarão de uma visita de iniciação do centro (treinamento on-line) antes do início do estudo para } \\
\text { garantir a consistência dos procedimentos, incluindo a coleta de dados. }\end{array}$ \\
\hline Contato & $\begin{array}{l}\text { Os pesquisadores poderão ligar para o Centro Coordenador do Estudo para resolver questões ou problemas que possam } \\
\text { surgir. }\end{array}$ \\
\hline Limpeza dos dados & $\begin{array}{l}\text { Será realizada limpeza periódica dos dados para identificar inconsistências (aproximadamente a cada 15 dias). Os centros } \\
\text { serão notificados de quaisquer inconsistências para fornecer correções. }\end{array}$ \\
\hline Validação estatística & $\begin{array}{l}\text { Técnicas estatísticas para a identificação de erros serão realizadas durante o estudo. Essas análises incluirão a identificação } \\
\text { de dados ausentes, dados inconsistentes, desvios de protocolos eventos incorretamente (dados } \\
\text { considerados inconsistentes com a revisão médica centralizada) e avaliação sistemática de erros. }\end{array}$ \\
\hline Revisão dos dados & $\begin{array}{l}\text { O Centro Coordenador/Patrocinador irá revisar os relatórios detalhados sobre triagem, inclusão, seguimento, consistência e } \\
\text { total preenchimento dos dados mensalmente e tomará medidas imediatas para resolver quaisquer problemas. }\end{array}$ \\
\hline
\end{tabular}




\begin{tabular}{|c|c|c|c|c|c|}
\hline \multirow[b]{3}{*}{ MOMENTOS } & \multicolumn{5}{|c|}{ Período do estudo } \\
\hline & \multirow{2}{*}{$\frac{\text { Recrutamento }}{-t_{1}}$} & \multirow{2}{*}{$\begin{array}{c}\text { Alocação } \\
0\end{array}$} & \multicolumn{2}{|c|}{ Pós-alocação } & \multirow{2}{*}{$\frac{\text { Término }}{t_{x}}$} \\
\hline & & & $t_{1}$ & $t_{2}$ & \\
\hline \multicolumn{6}{|l|}{ Recrutamento: } \\
\hline Triagem/elegibilidade & $x$ & & & & \\
\hline Consentimento informado & $x$ & $x$ & & & \\
\hline Alocação & & $X$ & & & \\
\hline \multicolumn{6}{|l|}{ INTERVENÇÕES } \\
\hline \multicolumn{6}{|l|}{ Hidroxicloroquina } \\
\hline \multicolumn{6}{|l|}{ Placebo } \\
\hline \multicolumn{6}{|l|}{ MEDIÇÕES } \\
\hline Basal & $x$ & $x$ & & & \\
\hline Desfechos & & & $x$ & $x$ & \\
\hline
\end{tabular}

Figura 2 - Esquema de coleta de dados e seguimento dos participantes.

\section{Análise estatística}

\section{Cálculo amostral}

Presume-se que o desfecho primário (hospitalização) ocorrerá em $20 \%$ dos indivíduos do grupo placebo e $14 \%$ dos indivíduos do grupo HCQ, correspondendo a uma redução de $30 \%$ no risco relativo. Essa premissa foi baseada na experiência clínica local nos primeiros meses da pandemia, em que $20 \%$ dos pacientes que não receberam nenhuma intervenção foram hospitalizados após o atendimento médico inicial. A escolha do efeito do tratamento (redução de $30 \%$ no risco relativo) baseou-se em plausibilidade razoável, com a maioria dos benefícios consistindo em efeitos moderados de tratamento (20$30 \%$ ). Estimou-se que uma amostra de 1.230 pacientes (615 por grupo) forneceria um poder estatístico de $80 \%$ para detectar essa redução a um nível de significância estatística de 5\% com o teste do qui-quadrado, premissa de hipótese de significância bilateral e alocação de 1:1. Foi estimada uma taxa de desistência de 5\% em cada grupo, o que resultaria em 1.296 indivíduos no total (648 por grupo). Portanto, decidiu-se que 1.300 indivíduos (650 por grupo) seriam randomizados para o estudo. O cálculo amostral foi realizado no software SAS 9.4 (procedimento PROC POWER). ${ }^{16,17}$

\section{Populações do estudo}

A avaliação do desfecho primário (hospitalização em 30 dias) será realizada de acordo com o princípio da intenção de tratar (intention-to-treat, ITT), que consistirá em todos os casos randomizados. Uma análise por ITT modificada (mITT) também será realizada após a exclusão de casos com teste negativo para covid-19.

\section{Estatísticas descritivas gerais}

Serão realizadas análises estatísticas após a resolução de todas as inconsistências, o controle de qualidade de dados e o bloqueio do banco de dados. As características clínicas e demográficas basais serão expressas em contagem e porcentagem, média e desvio padrão ou mediana e intervalo interquartil, conforme o caso. As análises de segurança irão considerar os desfechos de segurança, e os participantes serão classificados em grupos de tratamento de acordo com o medicamento que de fato receberam. Os motivos para a interrupção do estudo serão listados para cada grupo de tratamento.

A incidência de eventos adversos será resumida e apresentada por grupo de tratamento. Os eventos adversos serão resumidos de acordo com a gravidade e a intensidade por grupo de tratamento. Eventos adversos graves ou que levem à interrupção do tratamento serão listados por participante. O teste do qui-quadrado ou o teste exato de Fisher será utilizado para comparar os eventos adversos entre os dois grupos.

\section{Análise do desfecho primário}

O efeito da intervenção no desfecho primário e nos desfechos secundários binários será estimado por razão de 
risco (RR) e intervalo de confiança de 95\% (IC95\%). O teste do qui-quadrado ou o teste exato de Fisher será utilizado para os testes de hipótese. O mesmo procedimento será realizado para os desfechos secundários definidos por proporções.

O desfecho primário também será avaliado por meio de um modelo de regressão logística mista composto por um modelo de efeitos mistos com o grupo randomizado como efeito fixo e o centro como efeito aleatório. Será relatada a razão de chance com o IC95\%.

\section{Análise dos desfechos secundários}

Os desfechos secundários definidos por variáveis quantitativas de distribuição assimétrica e normal serão comparados entre os dois grupos (HCQ e placebo) através do teste $t$ de Student não pareado. O teste de Mann-Whitney será utilizado para as variáveis com distribuição não normal.

O modelo de regressão de Cox será utilizado para avaliar o efeito da intervenção na mortalidade aos 30 dias. Caso ocorra o fenômeno de probabilidade monótona, ou seja, observado um número raro de eventos, será aplicada a abordagem de probabilidade parcial penalizada de Firth no modelo de regressão de Cox univariado.

O modelo de regressão de Cox univariado será utilizado para avaliar o efeito da intervenção na sobrevida livre de hospitalizações aos 30 dias. A sobrevida livre de hospitalizações aos 30 dias será construída com o método de Kaplan-Meier, e o teste de log-rank será utilizado para avaliar as diferenças entre as curvas. Será relatada a razão de risco com o IC95\%.

As hipóteses de riscos proporcionais serão verificadas através de somas cumulativas de resíduos de Martingale e o teste supremo do tipo Kolmogorov com base em uma amostra de 1.000 padrões de resíduos simulados. ${ }^{18}$

\section{Análises interinas}

Três análises interinas serão realizadas utilizando a abordagem de Haybittle-Peto para avaliar a segurança e a eficácia do estudo, especificamente quando o tamanho amostral atingir 25\% (325 participantes), 50\% (650 participantes) e $75 \%$ (975 participantes). ${ }^{19}$ A segurança será avaliada nos dias 7 e 30, e a eficácia, no dia 30, em blocos separados. Quanto à regra de decisão, na avaliação de segurança, o estudo pode ser interrompido, de acordo com o método de Haybittle-Peto, caso haja sinal de dano (arritmia cardíaca grave, morte súbita, retinopatia em 7 dias) com $\mathrm{p}<0,01$ (em cada análise interina). A porcentagem de pacientes a serem analisados na avaliação de segurança no dia 7 é de $25 \%$, correspondendo a 325 participantes. Conforme descrito anteriormente, prevê-se a análise interina nesse momento. Na avaliação de eficácia, o estudo pode ser interrompido, de acordo com o método Haybittle-Peto, caso haja sinal de benefício (desfecho primário em 30 dias após a randomização) com $\mathrm{p}<0,001$ (em cada análise interina).

O limite de Haybittle-Peto é uma regra de interrupção conservadora na análise interina que possui um impacto mínimo no aumento de erros tipo I em ensaios com dois braços. ${ }^{20}$ Não haverá ajustes no limite final para significância estatística para análise sequencial.
Prevê-se que, durante as três análises interinas, a proporção entre casos negativos confirmados e aqueles que não foram testados será avaliada para estimar a necessidade de recálculo da amostra para garantir um poder estatístico adequado. De acordo com a proporção de casos não positivos de covid-19, será considerada a substituição da amostra, de forma a garantir um poder estatístico de $80 \%$ na amostra efetivamente analisada para o desfecho primário.

As análises serão realizadas a partir de dados completos. Além disso, será relatada a proporção de indivíduos não testados em cada grupo.

Todas as análises interinas pré-especificadas foram realizadas por um DSMB independente, que recomendou a continuação do estudo conforme o planejado após a realização de análises formais confidenciais e o envio de cartas oficiais ao Comitê Diretor do estudo COPE.

\section{Sensibilidade e análise de subgrupos}

As análises exploratórias para o desfecho primário serão realizadas considerando o efeito da intervenção dentro de subgrupos pré-especificados através de análises estratificadas e testes de interação. Esses testes de interação serão baseados em modelos de regressão logística binária que incluem o efeito do tratamento, o fator de interesse e um termo de interação entre as duas variáveis, com relato do valor de p para o termo de interação.

As análises serão conduzidas pelo princípio de análise de casos completos. Além disso, para o desfecho primário, se a proporção de dados ausentes for maior que 5\%, uma análise de sensibilidade será realizada através de técnica de imputação de dados múltiplos. ${ }^{21,22}$

Todos os testes de hipótese serão bilaterais. Um valor de $p<0,05$ será considerado estatisticamente significativo em todas as análises. As análises serão realizadas no software SAS, versão 9.4 (SAS Institute Inc, Cary, NC, EUA).

\section{Bloqueio da base de dados}

Será realizado o bloqueio da base de dados após a conclusão do seguimento de 30 dias de todos os pacientes. A limpeza dos dados será feita após o monitoramento clínico. Todas as análises interinas serão disponibilizadas às agências reguladoras locais do Brasil. Será concedido acesso à base de dados apenas aos membros do Comitê Diretor e aos estatísticos antes da publicação dos resultados principais.

\section{Supervisão do ensaio clínico}

O Comitê Executivo/Diretor é responsável pela supervisão geral do estudo, pelo desenvolvimento do protocolo de estudo e pela redação do manuscrito. Todos os demais comitês respondem ao Comitê Executivo/Diretor.

O DSMB irá avaliar os efeitos da HCQ comparada ao cuidado usual em relação ao desfecho primário (hospitalização em 30 dias) e à ocorrência de eventos adversos (em 7 e 30 dias) com necessidade de atendimento médico e/ou hospitalização. As regras para a interrupção precoce do estudo serão aplicadas ao objetivo primário (eficácia) e aos eventos 
adversos (segurança). O Comitê irá monitorar qualquer evento adverso grave e, se necessário, recomendar a interrupção do tratamento para garantir a segurança do paciente.

\section{Ética e disseminação}

Os registros dos participantes serão mantidos em sigilo e acessados de forma restrita apenas por indivíduos formalmente ligados ao estudo, que irão transferir as informações clínicas para formulários específicos (que não contêm informações que possam identificar os pacientes) e verificar se o estudo está sendo conduzido de forma adequada. O formulário eletrônico de coleta de dados incluirá a ID do participante e a ID do centro correspondente. Após a explicação completa dos riscos/ benefícios e dos procedimentos do estudo, todos os pacientes ou representantes legais devem assinar um TCLE em conformidade com as exigências locais.

O estudo foi aprovado pela Comissão Nacional de Ética em Pesquisa (CONEP) e pela Agência Nacional de Vigilância Sanitária (ANVISA). Está registrado no Registro Brasileiro de Ensaios Clínicos (REBEC) sob o número RBR-3cbs3w e no clinictrial.gov sob o código NCT04466540. Quaisquer alterações feitas no protocolo devem ser aprovadas pelo comitê de ética em pesquisa/sistema CONEP antes da implementação pelos centros participantes.

O pesquisador e a equipe do centro conduzirão o estudo de acordo com os princípios da Declaração de Helsinki, seguindo os princípios da boa prática clínica e todas as políticas e procedimentos regulatórios e internos aplicáveis.

\section{Referências}

1. $\mathrm{Xu} \mathrm{XW,} \mathrm{Wu} \mathrm{XX,} \mathrm{Jiang} \mathrm{XG,} \mathrm{Xu} \mathrm{KJ,} \mathrm{Ying} \mathrm{LJ,} \mathrm{Ma} \mathrm{CL,} \mathrm{et} \mathrm{al.} \mathrm{Clinical} \mathrm{Findings} \mathrm{in} \mathrm{a}$ Group of Patients Infected with the 2019 Novel Coronavirus (SARS-Cov-2) Outside of Wuhan, China: Retrospective Case Series. BMJ. 2020;368:m606. doi: 10.1136/bmj.m606

2. World Health Organization. Clinical Management of Severe Acute Respiratory Infection When Novel Coronavirus ( $\mathrm{nCoV}$ ) Infection is Suspected. Geneva: WHO Library; 2020.

3. Huang C, Wang Y, Li X, Ren L, Zhao J, Hu Y, et al. Clinical Features of Patients Infected with 2019 Novel Coronavirus in Wuhan, China. Lancet. 2020;395(10223):497-506. doi: 10.1016/S0140-6736(20)30183-5.

4. Li B, Yang J, Zhao F, Zhi L, Wang X, Liu L, et al. Prevalence and Impact of Cardiovascular Metabolic Diseases on COVID-19 in China. Clin Res Cardiol. 2020;109(5):531-38. doi: 10.1007/s00392-020-01626-9.

5. Abella BS, Jolkovsky EL, Biney BT, Uspal JE, Hyman MC, Frank I, et al. Efficacy and Safety of Hydroxychloroquine vs Placebo for Pre-Exposure SARS-CoV-2 Prophylaxis Among Health Care Workers: A Randomized Clinical Trial. JAMA Intern Med. 2021;181(2):195-202. doi: 10.1001/ jamainternmed.2020.6319.

6. Rajasingham R, Bangdiwala AS, Nicol MR, Skipper CP, Pastick KA, Axelrod $\mathrm{ML}$, et al. Hydroxychloroquine as Pre-exposure Prophylaxis for Coronavirus Disease 2019 (COVID-19) in Healthcare Workers: A Randomized Trial. Clin Infect Dis. 2021;72(11):835-43. doi: 10.1093/cid/ciaa1571.

7. Barnabas RV, Brown ER, Bershteyn A, Stankiewicz Karita HC, Johnston C, et al. Hydroxychloroquine as Postexposure Prophylaxis to Prevent Severe Acute Respiratory Syndrome Coronavirus 2 Infection: A Randomized Trial. Ann Intern Med. 2021 Mar;174(3):344-52. doi: 10.7326/M20-6519.
Após a conclusão, o estudo será submetido para publicação independentemente dos resultados e será divulgado conforme solicitado pelas autoridades locais.

\section{Contribuição dos autores}

Concepção e desenho da pesquisa: Oliveira Junior HA, Ferri CP, Boszczowski I, Oliveira GBF, Rosa RG, Lopes RD, Azevedo LCP, Veiga VC, Berwanger O, Avezum A, Cavalcanti AB; Obtenção de dados: Oliveira Junior HA, Boszczowski I, Oliveira GBF, Avezum A; Análise e interpretação dos dados: Oliveira Junior HA, Oliveira GBF, Avezum A; Obtenção de financiamento: Oliveira Junior HA, Oliveira GBF, Avezum A; Redação do manuscrito: Oliveira Junior HA, Ferri CP, Oliveira GBF, Avezum A; Revisão crítica do manuscrito quanto ao conteúdo intelectual importante: Oliveira Junior $\mathrm{HA}$, Ferri CP, Oliveira GBF, Rosa RG, Lopes RD, Azevedo LCP, Veiga VC, Berwanger O, Avezum A, Cavalcanti AB.

\section{Potencial conflito de interesse}

Não há conflito com o presente artigo

\section{Fontes de financiamento}

O presente estudo foi parcialmente financiado por E.M.S Farmacêutica.

\section{Vinculação acadêmica}

Não há vinculação deste estudo a programas de pós-graduação.

8. Boulware DR, Pullen MF, Bangdiwala AS, Pastick KA, Lofgren SM, Okafor EC, et al. A Randomized Trial of Hydroxychloroquine as Postexposure Prophylaxis for Covid-19. N Engl J Med. 2020;383(6):517-25. doi: 10.1056/ NEJMoa2016638.

9. Mitjà $\mathrm{O}$, Corbacho-Monné $\mathrm{M}$, Ubals $\mathrm{M}$, Alemany A, Suñer C, Tebé C, et al. A Cluster-Randomized Trial of Hydroxychloroquine for Prevention of Covid-19. N Engl J Med. 2021;384(5):417-27. doi: 10.1056/ NEJMoa2021801.

10. Mitjà $\mathrm{O}$, Corbacho-Monné $M$, Ubals $M$, Tebé $C$, Peñafiel J, Tobias $A$, et al. Hydroxychloroquine for Early Treatment of Adults With Mild Coronavirus Disease 2019: A Randomized, Controlled Trial. Clin Infect Dis. 2021;73(11):4073-81. doi: 10.1093/cid/ciaa1009.

11. Skipper CP, Pastick KA, Engen NW, Bangdiwala AS, Abassi M, Lofgren SM, et al. Hydroxychloroquine in Nonhospitalized Adults with Early COVID-19: A Randomized Trial. Ann Intern Med. 2020;173(8):623-31. doi: 10.7326/ M20-4207

12. Omrani AS, Pathan SA, Thomas SA, Harris TRE, Coyle PV, Thomas CE, et al. Randomized Double-Blinded Placebo-Controlled Trial of Hydroxychloroquine with or without Azithromycin for Virologic Cure of Non-Severe COVID-19. EClinicalMedicine. 2020;29:100645. doi: 10.1016/j.eclinm.2020.100645.

13. Brasil. Ministério da Saúde. Diretrizes para Diagnóstico e Tratamento da COVID-19 (Clinical Guideline Covid-19) [Internet]. Brasília: Ministério da Saúde. 2020 [cited 2021 Mar 18]. Available from: https:// portaldeboaspraticas.iff.fiocruz.br/atencao-mulher/diretrizes-paradiagnostico-e-tratamento-da-covid-19-ms/. 


\section{Artigo Original}

14. World Health Organization. Country \& Technical Guidance - Coronavirus Disease (COVID-19) [Internet]. Geneva: WHO. 2021 [cited 2021 Mar 18]. Available from: https://www.who.int/emergencies/diseases/novelcoronavirus-2019/technical-guidance.

15. Costa IBSDS, Bittar CS, Rizk SI, Araújo Filho AE, Santos KAQ, Machado TIV, et al. The Heart and COVID-19: What Cardiologists Need to Know. Arq Bras Cardiol. 2020;114(5):805-16. doi: 10.36660/abc.20200279.

16. Ryan TP. Sample Size Determination and Power. New Jersey: Wiley; 2013.

17. Shein-Chung C, Wang JSH. Sample Size Calculations in Clinical Research Second Edition (Chapman \& Hall/CRC Biostatistics Series). 2nd ed. Boca Raton: Chapman \& Hall/CRC Biostatistics Series; 2008.

18. Lachin JM, Matts JP, Wei LJ. Randomization in Clinical Trials: Conclusions and Recommendations. Control Clin Trials. 1988;9(4):365-74. doi: 10.1016/0197-2456(88)90049-9.
19. Haybittle JL. Repeated Assessment of Results in Clinical Trials of Cancer Treatment. Br J Radiol. 1971;44(526):793-7. doi: 10.1259/0007-128544-526-793.

20. Blenkinsop A, Parmar MK, Choodari-Oskooei B. Assessing the Impact of Efficacy Stopping Rules on the Error Rates Under the MultiArm Multi-Stage Framework. Clin Trials. 2019;16(2):132-41. doi: 10.1177/1740774518823551.

21. Raghunathan T, Berglund PA, Solenberger PW. Multiple Imputation in Practice: With Examples Using IVEware. Milton Park: Taylor \& Francis; 2018.

22. Patricia Berglund SGH. Multiple Imputation of Missing Data Using SAS. Cary: SAS Institute; 2014.

* Material suplementar

Para informação adicional, por favor, clique aqui. 